\title{
ALGEBRAIC $K$-THEORY OF PURE BRAID GROUPS*
}

\author{
C. S. ARAVINDA ${ }^{\dagger}$, F. T. FARRELL ${ }^{\ddagger}$, AND S. K. ROUSHON ${ }^{\S}$
}

\begin{abstract}
In this article we introduce the notion of strongly poly-free groups and show that the Whitehead group of any such group vanishes. We also show that the pure braid groups are strongly poly-free.
\end{abstract}

0. Introduction. A well-known conjecture in Geometry and Topology states that the Whitehead group of any finitely presented torsion free discrete group vanishes. So far this has been checked for many classes of such groups, e.g. free group [ST1], free abelian group [BHS], fundamental group of closed nonpositively curved Riemannian manifolds [FJ1]. There is a recent extension of the last result to complete nonpositively curved Riemannian manifold with some regularity condition on the sectional curvature [FJ3].

Now given any finitely presented torsion free group, to check the vanishing of the Whitehead group for this group, at first one would like to check if this group is the fundamental group of a complete nonpositively curved Riemannian manifold. We consider the case of pure braid group. It is not yet known if these groups can even act faithfully as a group of motions on a simply connected complete nonpositively curved Riemannian manifold. In this paper we introduce a new class of groups, namely the strongly poly-free groups which is a proper subclass of the class of poly-free groups and prove that the Whitehead group vanishes in this class of groups. We also show that the pure braid groups are strongly poly-free. We use some geometric results on 3-manifolds which fiber over the circle, the Fibered Isomorphism Theorem of Farrell and Jones and an induction argument to prove the result.

The paper is organized in the following way. In Section 1 we define strongly polyfree groups and state and prove that the Whitehead group of these groups are trivial. Section 2 is devoted to proving that the pure braid groups are strongly poly-free. We prove that the Whitehead group of the pure braid group of any compact (connected) surface except for the 2-sphere and the projective 2-plane vanishes in Section 3.

ACKNowledgment. This research was supported in part by the National Science Foundation and by the United Nations Educational, Scientific and Cultural Organization. The project crystallized while the authors were visitors at the Abdus Salam International Center for Theoretical Physics. They wish to thank ICTP for the kind invitation and hospitality which greatly facilitated the completion of this research. Part of this work was done during the last author's stay in the Max-Planck Institut für Mathematik. He would like to thank the institute for the hospitality.

1. A vanishing criterion and strongly poly-free groups. This section is devoted to defining the notion of strongly poly-free group and to proving that the Whitehead group of these groups vanishes.

\footnotetext{
* Received March 4, 1999; accepted for publication May 31, 1999.

† SPIC Mathematical Institute, 92, G. N. Chetty Road, Chennai 600 017, India (aravinda@smi. ernet.in).

$\ddagger$ Department of Mathematics, SUNY, Binghamton, NY 13902, USA.

$\S$ School of Mathematics, Tata Institute of Fundamental Research, Homi Bhabha Road, Mumbai 400 005, India (roushon@math.tifr.res.in).
} 
Throughout this section $T$ will denote the infinite cyclic group and $T^{m}$ the free abelian group of rank $m$.

We start with the definition of strongly poly-free group.

Definition 1.1. A discrete group $\Gamma$ is called strongly poly-free if there exists a finite filtration of $\Gamma$ by subgroups: $1=\Gamma_{0} \subset \Gamma_{1} \subset \cdots \subset \Gamma_{n}=\Gamma$ such that the following conditions are satisfied:

(1) $\Gamma_{i}$ is normal in $\Gamma$ for each $i$

(2) $\Gamma_{i+1} / \Gamma_{i}$ is a finitely generated free group

(3) for each $\gamma \in \Gamma$ and $i$ there is a compact surface $F$ and a diffeomorphism $f: F \rightarrow F$ such that the induced homomorphism $f_{\#}$ on $\pi_{1}(F)$ is equal to $c_{\gamma}$ in $\operatorname{Out}\left(\pi_{1}(F)\right)$, where $c_{\gamma}$ is the action of $\gamma$ on $\Gamma_{i+1} / \Gamma_{i}$ by conjugation and $\pi_{1}(F)$ is identified with $\Gamma_{i+1} / \Gamma_{i}$ via a suitable isomorphism.

In such a situation we say that the group $\Gamma$ has $\operatorname{rank} \leq n$.

Here we recall that $\Gamma$ is called poly-free if there is a finite filtration by subgroup satisfying condition (2) as above and $\Gamma_{i}$ is normal in $\Gamma_{i+1}$ for each $i$.

We denote the class of strongly poly-free groups by $\mathcal{S P \mathcal { F }}$.

REMARK 1.2. Here we remark that the class $\mathcal{S P F}$ is a proper subclass of the class of poly-free groups. Major difference between these two classes is the condition (3) in the definition of strongly poly-free group. This condition is in general not satisfied. For example in [ST2] Stallings gave example of an automorphism of a free nonabelian group which is not induced by a homeomorphism of a compact surface with fundamental group isomorphic to the given free group.

Now we state the main theorem of this article:

Theorem 1.3. The Whitehead group $W h\left(\Gamma \times T^{m}\right)=0$, for every $\Gamma \in \mathcal{S P F}$ and every nonnegative integer $m$.

We remark that a direct approach to proving Theorem 1.3 via Waldhausen's fundamental work in algebraic $K$-theory [W1] and [W2] is obstructed by the lack of a sufficiently strong vanishing theorem for his $N i$-groups.

The following corollary is immediate from Theorem 1.3.

Corollary 1.4. $K_{i}(\mathbb{Z} \Gamma)=0$ for all $i<0$ and $\tilde{K}_{0}(\mathbb{Z} \Gamma)=0$ for every $\Gamma \in \mathcal{S P \mathcal { F }}$.

The main tool to prove the theorem is the Fibered Isomorphism Conjecture of Farrell and Jones ([FJ2]). If we restrict to the functor of pseudo-isotopy spaces $\mathcal{P}_{*}()$ (in the notation of [FJ2]), then it is proved in [[FJ2], Proposition 2.3] that the conjecture is true for a connected $C W$-complex $X$ whose fundamental group acts properly discontinuously and co-compactly via isometries on a simply connected, symmetric nonpositively curved Riemannian manifold. As mentioned in the proof of Theorem A in [BFJP] this is also true if we replace "symmetric" by "complete" in the statement of Proposition 2.3 in [FJ2] and require $\pi_{1}(X)$ to be torsion-free. In the following proposition we state a version of this theorem for $C W$-complexes whose fundamental group is an npc-group (see Definition 1.5 for npc-group). The proof of this proposition follows from the above extension of Proposition 2.3 in [FJ2] and Theorem A.8 and Corollary 2.2.1 in [FJ2] and by noting the fact that any npc-group is a subgroup of the fundamental group of a closed nonpositively curved Riemannian manifold.

Before we state the proposition we need the following definition.

Definition 1.5. A group $\pi$ is called an npc-group if it is the fundamental group 
of a complete nonpositively curved connected Riemannian manifold $M$ and there is a compact subset $K$ of $M$ such that $M-K$ has finitely many components and each component is isometric to a product $(0, \infty) \times N$.

Proposition 1.6. Let $\pi$ be an npc-group and let $p: G \rightarrow \pi$ be a surjective homomorphism from a group $G$. Suppose that $W h\left(p^{-1}(C) \times T^{m}\right)=0$ for every nonnegative integer $m$ and every cyclic subgroup $C$ of $\pi$ (including $C=1$ ). Then $W h(G)=0$.

Obviously the fundamental group of any closed oriented surface of genus bigger than 0 and any finitely generated free group are examples of npc-groups. Below we give some more examples of npc-group which we need for the proof of the Theorem 1.3 .

Let $\mathcal{F}$ be a finitely generated free group and $\gamma: \mathcal{F} \rightarrow \mathcal{F}$ be an automorphism. Suppose the automorphism $\gamma$ is induced by a diffeomorphism $f: F \rightarrow F$, where $F$ is a compact 2-manifold with boundary and $\pi_{1}(F)$ is isomorphic to $\mathcal{F}$. Then we prove the following lemma:

LEMMA 1.7. The semidirect product $\mathcal{F} \rtimes T$, where $T$ is the infinite cyclic group and it is acting on $\mathcal{F}$ via the automorphism $\gamma$, is the fundamental group of a compact 3-manifold which supports on its interior a complete nonpositively curved Riemannian metric and the metric is a cylinder near the boundary.

Proof of Lemma 1.7. Consider the mapping torus of the diffeomorphism $f: F \rightarrow$ $F$, say $M$. Clearly $\pi_{1}(M)=\mathcal{F} \rtimes T$ and $M$ is a compact 3-manifold with nonempty boundary, whose components are tori or Klein bottles. Since the universal cover of the interior of $M$ is homeomorphic to Euclidean 3-space, $M$ is irreducible. Now it is a standard fact that an irreducible 3-manifold with nonempty boundary is Haken. Hence the proof of the lemma follows from the main theorem in [L].

Proof of Theorem 1.3. We prove the theorem by induction on the rank of the group $\Gamma$. The $n$-th induction statement is that $W h\left(\Gamma \times T^{m}\right)=0$ for all nonnegative integer $m$ and every $\Gamma \in \mathcal{S P F}$ of rank $\leq n$. Induction statement 1 is true since in this case the group $\Gamma$ is finitely generated free and the Proposition 1.6 is applicable to the homomorphism $p: \Gamma \times T^{m} \rightarrow \Gamma$.

Assuming the $n$-th induction statement is true we proceed to deduce the $(n+1)$ th induction statement. We start by applying the Proposition 1.6 to the composite homomorphism $p: \Gamma \times T^{m} \rightarrow \Gamma \rightarrow \Gamma / \Gamma_{n}=\pi$. Note that $\pi$ is an npc-group and let $q: \Gamma \rightarrow \Gamma / \Gamma_{n}=\pi$ denote the canonical surjection.

Case 1: Suppose $C=1$ in Proposition 1.6 then $p^{-1}(C)=\Gamma_{n} \times T^{m}$ and $W h\left(\Gamma_{n} \times\right.$ $\left.T^{m}\right)=0$ since $\Gamma_{n}$ is strongly poly-free of rank $\leq n$.

Case 2: Assume $C$ is infinite cyclic. Then $p^{-1}(C)=q^{-1}(C) \times T^{m}$. Now we apply Proposition 1.6 to the composite $p_{1}: q^{-1}(C) \times T^{m} \rightarrow q^{-1}(C) \rightarrow q^{-1}(C) / \Gamma_{n-1}$.

Claim. $q^{-1}(C) / \Gamma_{n-1}$ is an npc-group.

We will prove the claim during the end of this proof.

Let $C_{1}$ be a cyclic subgroup of $q^{-1}(C) / \Gamma_{n-1}$. If $C_{1}=1$ then $p_{1}^{-1}\left(C_{1}\right)=\Gamma_{n-1} \times$ $T^{m}$. Hence $p_{1}^{-1}\left(C_{1}\right) \times T^{m_{1}}=\Gamma_{n-1} \times T^{m+m_{1}}$ and $W h\left(\Gamma_{n-1} \times T^{m+m_{1}}\right)=0$ since $\Gamma_{n-1}$ is strongly poly-free group of rank $\leq n-1$. Now assume $C_{1}$ is infinite cyclic. Consider the projection $q_{1}: \Gamma \rightarrow \Gamma / \Gamma_{n-1}$. Under this projection $q^{-1}(C)$ goes onto $q^{-1}(C) / \Gamma_{n-1}$. Thus we have $p_{1}^{-1}\left(C_{1}\right)=q_{1}^{-1}\left(C_{1}\right) \times T^{m}$ and hence $p_{1}^{-1}\left(C_{1}\right) \times T^{m}=q_{1}^{-1}\left(C_{1}\right) \times T^{m+m_{1}}$. Now note that the filtration $1=\Gamma_{0} \subset \Gamma_{1} \subset \cdots \subset \Gamma_{n-1} \subset q_{1}^{-1}\left(C_{1}\right)$ displays $q_{1}^{-1}\left(C_{1}\right)$ 
as a strongly poly-free group of rank $\leq n$. Therefore by the induction hypothesis $W h\left(p_{1}^{-1}\left(C_{1}\right) \times T^{m_{1}}\right)=0$.

Hence applying the Proposition 1.6 to $p_{1}$ yields $W h\left(q^{-1}(C) \times T^{m}\right)=0$.

Therefore the proposition can be applied again to $p$ to complete the proof of the theorem, i.e., $W h\left(\Gamma \times T^{m}\right)=0$.

Now we come back to the claim we made above:

Proof of the claim. We have $q^{-1}(C) / \Gamma_{n-1}=\frac{\Gamma_{n}}{\Gamma_{n-1}} \rtimes C$, where the generator of $C$ acts on $\Gamma_{n} / \Gamma_{n-1}$ by conjugation. If $\Gamma_{n} / \Gamma_{n-1}=1$ or $\mathbb{Z}$, then $q^{-1}(C) / \Gamma_{n-1}$ is either $\mathbb{Z}$ or $\mathbb{Z}^{2}$ or the fundamental group of the Klein bottle. In all cases it is an npc-group. So assume $\Gamma_{n} / \Gamma_{n-1}$ is a nonabelian free group. Now from the definition of strongly poly-free group and by Lemma 1.7 it follows that $q^{-1}(C) / \Gamma_{n-1}$ is an npc-group.

2. Classical pure braid groups. In this section we show that the classical pure braid groups belong to the class $\mathcal{S P \mathcal { F }}$.

We start with some notations and recall the definition of pure braid group.

Let $P_{n}=\{1,2, \ldots, n\} \subset \mathbb{C}$, here $\mathbb{C}$ denotes the complex plane.

Define $M_{n}^{k}=\left\{\left(x_{1}, x_{2}, \ldots, x_{n}\right) \mid x_{i} \in \mathbb{C}-P_{k}\right.$ for each $i$ and $x_{i} \neq x_{j}$ for $\left.i \neq j\right\}$. Then the pure braid group $B_{n}$ of $n$ strands is by definition the fundamental group of the space $M_{n}^{0}$. Note that the projection $M_{m}^{j} \rightarrow M_{k}^{j}$ to the first $k$ coordinates is a fiber bundle projection with fiber diffeomorphic to $M_{m-k}^{k+j}$. Using these fiber bundles and the homotopy exact sequence of a fibration it follows that the spaces $M_{m}^{j}$ are aspherical, i.e., $\pi_{i}\left(M_{m}^{j}\right)=0$ for $i>1$ and hence $B_{n}$ is torsion free.

The main theorem of this section is the following.

THEOREM 2.1. The pure braid group $B_{n}$ is strongly poly-free of rank $\leq n$.

Here we point out some immediate consequences of Theorem 2.1 and Theorem 1.3:

THEOREM 2.2. $W h\left(B_{n} \times T^{m}\right)=0$ for all nonnegative integer $m$ and for $n \geq 1$.

Corollary 2.3. $\tilde{K}_{0}\left(\mathbb{Z} B_{n}\right)=0$ and $K_{-i}\left(\mathbb{Z} B_{n}\right)=0$ for all $n, i \geq 1$.

Before giving the proof of the theorem we note some generalization of the pure braid group and state a natural conjecture. From the above description we see that the pure braid group is the fundamental group of the complement of the arrangements of hyperplanes $\left\{x_{i}=x_{j}\right\}$, for $i, j \in\{1,2, \ldots, n\}$ and $i \neq j$ in the complex $n$-space $\mathbb{C}^{n}$. Also it is clear that this complement sits at the top of a tower of fiber bundles:

$$
M_{n}^{0} \rightarrow M_{n-1}^{0} \rightarrow \cdots \rightarrow M_{2}^{0} \rightarrow M_{1}^{0}
$$

and at each stage the fiber is the complex plane minus finitely many points. An obvious generalization of this particular type of hyperplane arrangement is the complement of hyperplanes in the complex $n$-space which has the property that after a linear change of coordinates in $\mathbb{C}^{n}$ the complements sits at the top of a tower of fiber bundles with the above property. These arrangements are known as fiber-type arrangements. Obviously the complement of any fiber-type arrangement is aspherical. As we remarked before the example (by Stallings) of an automorphism of a free group which is topologically unrealizable, it is unlikely that the fundamental group of every fiber-type hyperplane arrangement complement is strongly poly-free. But the method of the proof of the Theorem 1.3 suggests the following conjecture: 
CONJECTURE ${ }^{1}$. Wh $\left(\pi_{1}(M) \times T^{m}\right)=0$ for any nonnegative integer $m$, where $M$ is the complement of a fiber-type hyperplane arrangement in the complex $n$-space.

To prove Theorem 2.1 we need an alternative description of the pure braid group.

Let $E\left(P_{n}, \mathbb{C}\right)=$ space of all embeddings of $P_{n}$ in $\mathbb{C}$. There is an identification of $E\left(P_{n}, \mathbb{C}\right)$ with $M_{n}^{0}$. Hence $B_{n}=\pi_{1}\left(E\left(P_{n}, \mathbb{C}\right)\right)$. Also note that a path in $E\left(P_{n}, \mathbb{C}\right)$ is an isotopy of embeddings and a loop is an isotopy of the inclusion $P_{n} \subset \mathbb{C}$ back to itself.

Proof of Theorem 2.1. We have to give a filtration of $B_{n}$ such that the conditions (1)-(3) are satisfied.

We start with the fiber bundle projection $M_{m}^{j} \rightarrow M_{k}^{j}$ which is given by projecting to the first $k$ coordinates: $\left(x_{1}, x_{2}, \ldots, x_{m}\right) \rightarrow\left(x_{1}, x_{2}, \ldots, x_{k}\right)$. The fiber of this fiber bundle is diffeomorphic to $M_{m-k}^{k+j}$. From the homotopy exact sequence of a fibration and by an induction argument it follows that each $M_{m}^{j}$ is aspherical. Hence we have an exact sequence:

$$
1 \rightarrow \pi_{1}\left(M_{m-k}^{k+j}\right) \rightarrow \pi_{1}\left(M_{m}^{j}\right) \rightarrow \pi_{1}\left(M_{k}^{j}\right) \rightarrow 1
$$

We define a filtration of $B_{n}$ in the following way: $1=\Gamma_{0} \subset \Gamma_{1} \subset \cdots \subset \Gamma_{n}=B_{n}$, where $\Gamma_{i}=\pi_{1}\left(M_{i}^{n-i}\right)$. From the above exact sequence it is clear that $\Gamma_{i}$ is normal in $\Gamma_{n}=B_{n}$ for each $i$. So we have checked that a filtration of $B_{n}$ exists satisfying condition (1) in the definition of strongly poly-free group.

Now we proceed to check that condition (2) is also satisfied. This follows from the fiber bundle projection $M_{i+1}^{n-(i+1)} \rightarrow M_{1}^{n-(i+1)}$. The fiber of this fiber bundle is $M_{i}^{n-i}$. Clearly $\Gamma_{i+1} / \Gamma_{i}=\pi_{1}\left(M_{1}^{n-(i+1)}\right)$ is a free group on $n-(i+1)$ generators, since $M_{1}^{n-(i+1)}=\mathbb{C}-P_{n-(i+1)}$.

The burden of the argument is to check the condition (3) in the definition of strongly poly-free group. For this we use the alternative description of $B_{n}$ as the fundamental group of the space $E\left(P_{n}, \mathbb{C}\right)$ as described in the beginning of this section.

First we fix a representative $\gamma$ of some element $[\gamma] \in B_{n}$. We regard the element $\gamma$ as an isotopy $\gamma_{t}, t \in[0,1]$ of the inclusion $P_{n} \subset \mathbb{C}$ back to itself. Hence we can apply the isotopy extension theorem to get an ambient isotopy $\tilde{\gamma}_{t}: \mathbb{C} \rightarrow \mathbb{C}, t \in[0,1]$ extending $\gamma_{t}$ and starting at $i d_{\mathbb{C}}$, i.e., $\tilde{\gamma}_{0}=i d_{\mathbb{C}} ; \tilde{\gamma}_{t}(j)=\gamma_{t}(j)$ for all $t \in[0,1]$ and for all $j=1,2, \ldots, n$.

We will be particularly interested in $\tilde{\gamma}_{1}$ which we call the monodromy of the braid $\gamma$. Note that $\tilde{\gamma}_{1}$ leaves $S=\mathbb{C}-P_{n-i}$ invariant and hence we define the diffeomorphism $f: S \rightarrow S$ to be the restriction of $\tilde{\gamma}_{1}$ to $\mathbb{C}-P_{n-i}$. Here we note that the surface $S$ is diffeomorphic to the extended complex plane $\hat{\mathbb{C}}$ punctured at the points $1,2, \ldots n-i, \infty$ and $f$ is a diffeomorphism of $\hat{\mathbb{C}}$ fixing these punctures. Now we can again isotope $f$ to a diffeomorphism which fixes some disc neighborhood of each of these punctures and hence restricts to a diffeomorphism $g$ of a compact surface. Also $f$ and $g$ induce the same outer automorphism of $\pi_{1}(S)$. We already observed that $\pi_{1}(S)=\Gamma_{i} / \Gamma_{i-1}$ and hence it remains to show that $f_{\#}=c_{[\gamma]}$ in $\operatorname{Out}\left(\pi_{1}(S)\right)$. Recall $c_{[\gamma]}$ is by definition the conjugation action by $[\gamma]$.

Note that $\tilde{\gamma}_{1}$ naturally induces diffeomorphisms $f_{j}^{k}: M_{j}^{k} \rightarrow M_{j}^{k}$ for all nonnegative integers $j$ and $k$ with $j+k \leq n$ by the formula $f_{j}^{k}\left(x_{1}, x_{2}, \ldots, x_{j}\right)=\left(\tilde{\gamma}_{1}\left(x_{1}\right), \tilde{\gamma}_{1}\left(x_{2}\right)\right.$,

\footnotetext{
${ }^{1}$ Recently this conjecture is proved in $[F R]$ and also independently by D. Cohen in [C].
} 
$\left.\ldots, \tilde{\gamma}_{1}\left(x_{j}\right)\right)$ which gives bundle self maps to each of the bundles $M_{j}^{k} \rightarrow M_{i}^{k}$ provided $j+k \leq n$. We will presently use this to prove the following assertion.

Claim. $\left(f_{i}^{n-i}\right)_{\#}=c_{[\gamma]}$ in $\operatorname{Out}\left(\Gamma_{i}=\pi_{1}\left(M_{i}^{n-i}\right)\right)$.

Before verifying the claim we use it to complete the proof of Theorem 2.1.

We have the fiber bundle projection: $p: M_{i}^{n-i} \rightarrow S$. Recall that $S=M_{1}^{n-i}=$ $\mathbb{C}-P_{n-i}$. Now the following three facts together with the claim above show directly that $f_{\#}=c_{[\gamma]}$ in $\operatorname{Out}\left(\pi_{1}(S)\right)$ :

1. $p_{\#}$ is a surjective homomorphism.

2. $p_{\#} \circ c_{[\gamma]}=c_{[\gamma]} \circ p_{\#}$ after identifying $p_{\#}$ with the canonical quotient map $\Gamma_{i} \rightarrow \Gamma_{i} / \Gamma_{i-1}$.

3. $p_{\#} \circ\left(f_{i}^{n-i}\right)_{\#}=\left(f_{1}^{n-i}\right)_{\#} \circ p_{\#}$.

It remains to verify the claim. For this purpose we need the following lemma whose proof is left to the reader.

Before we state the lemma we need some terminology. Let $\phi: F \rightarrow F$ be a self homeomorphism. Let $F_{\phi}$ denotes the mapping torus of $\phi$, i.e., the quotient space of $F \times[0,1]$ where $(x, 1)$ is identified with $(\phi(x), 0)$ and the projection onto the second factor induces a fiber bundle projection: $q: F_{\phi} \rightarrow \mathbb{S}^{1}$.

LemMA 2.4. Let $p: E \rightarrow M$ be a fiber bundle with arc connected fiber $F$ and $\phi: F \rightarrow F$ be a self homeomorphism. Let $g: F_{\phi} \rightarrow E$ be a bundle map covering $\alpha: \mathbb{S}^{1} \rightarrow M$ and $\bar{\alpha}: \mathbb{S}^{1} \rightarrow E$ be a lift of $\alpha$. Let $[\bar{\alpha}] \in \pi_{1}(E)$ denote the homotopy class of $\bar{\alpha}$. Then $c_{[\bar{\alpha}]}=\phi_{\#}$ in Out $\left(\pi_{1}(F)\right)$ provided $\pi_{2}(M)=0$ and the fiber containing the base point of $E$ is identified with $F$ via $g$.

The claim directly follows from the Lemma 2.4 and by setting $p: E \rightarrow M$ to be the following bundle projection: $M_{n}^{0} \rightarrow M_{n-i}^{0}$ and $F=M_{i}^{n-i}$ and $\phi=f_{i}^{n-i}$. Let $\bar{\alpha}=\gamma$ be a fixed representative of the homotopy class $[\gamma] \in B_{n}=\pi_{1}(E)$ and $\alpha=p \circ \bar{\gamma}$. Define

$$
g\left(x_{1}, x_{2}, \ldots, x_{i}, t\right)=\left(\tilde{\gamma}_{t}(1), \tilde{\gamma}_{t}(2), \ldots, \tilde{\gamma}_{t}(n-i), \tilde{\gamma}_{t}\left(x_{1}\right), \tilde{\gamma}_{t}\left(x_{2}\right), \ldots, \tilde{\gamma}_{t}\left(x_{i}\right)\right)
$$

So we have proved that the condition (3) in the definition of strongly poly-free group is also satisfied for the filtration of pure braid group we defined before. Hence the proof of the theorem is complete.

3. Pure braid groups of surfaces. In this short section we prove that the Whitehead group of the pure braid groups of any compact (connected) surface $S$ except for the 2-sphere and the projective 2-plane vanishes.

We define the space $M_{n}^{0}(N)$ for any connected surface $N$ as the space $M_{n}^{0}$ we defined before, but we replace $\mathbb{C}$ by the space $N-\partial N$, where $\partial N$ is the boundary of $N$. The pure braid group of $N$ of $n$ strands is by definition the fundamental group of the space $M_{n}^{0}(N)$.

THEOREM 3.1. $W h\left(\pi_{1}\left(M_{n}^{0}(S)\right) \times T^{m}\right)=0$ for any nonnegative integer $m$ and for any $n \geq 1$, where $S$ is any compact (connected) surface (may be with nonempty boundary) other than $\mathbb{S}^{2}$ and $\mathbb{R P}^{2}$.

REMARK 3.2. Here we recall that the pure braid groups of (connected) surfaces are torsion free except for the cases $\mathbb{S}^{2}$ and $\mathbb{R P}^{2}$.

Proof of Theorem 3.1. We divide the proof in two cases: 
Case 1: Let $S$ be a compact (connected) surface with nonempty boundary. In this case it is easy to adapt the argument proving Theorem 2.1 to show that $\pi_{1}\left(M_{n}^{0}(S)\right)$ is a strongly poly-free group. Now the proof follows from Theorem 1.3.

Case 2: Assume $S$ is closed. Consider the fiber bundle projection $p: M_{n}^{0}(S) \rightarrow$ $M_{1}^{0}(S)=S$. The fiber of this fiber bundle is $M_{n-1}^{1}(S)=M_{n-1}^{0}(S-*)$ where $* \in S$. Note that $\pi_{1}\left(M_{n-1}^{1}(S)\right)$ is a strongly poly-free group. As $\pi_{1}(S)$ is an npc-group, we can use the Proposition 1.6. Let $C$ be a cyclic subgroup of $\pi_{1}(S)$, then $p_{\#}^{-1}(C)$ is a strongly poly-free group.

This proves the theorem.

\section{REFERENCES}

[BFJP] E. Berkove, F. T. Farrell, D. Juan-Pineda, and K. Pearson, The Farrell-Jones isomorphism conjecture for finite co-volume hyperbolic actions and the algebraic $K$ theory of Bianchi groups, to appear in Trans. Amer. Math. Soc..

[BHS] H. BASS, A. Heller, AND R. SwAN, The Whitehead group of a polynomial extension, Publ. Inst. Hautes Etudes Sci., 22 (1964), pp. 61-79.

[C] D. COHEN, Monodromy of fiber-type arrangements and orbit configuration space, to appear in Forum Math..

[FJ1] F. T. FARREll AND L. E. JonEs, Stable pseudoisotopy spaces of non-positively curved manifolds, Jour. of Diff. Geom., 34 (1991), pp. 769-834.

[FJ2] — Isomorphism conjectures in algebraic K-theory, J. Amer. Math. Soc., 6 (1993), pp. 249-297.

[FJ3] — Rigidity for aspherical manifolds with $\pi_{1} \subset G L_{m}(\mathbb{R})$, Asian J. Math., 2 (1998), pp. 215-262.

[FR] F. T. FARRELl AND SAYed K. Roushon, The Whitehead groups of braid groups vanish, Internat. Math. Res. Notices, 2000, no. 10, pp. 515-526.

[L] B. LEEB, 3-manifolds with(out) metrices of nonpositive curvature, Invent. Math., 122 (1995), pp. 277-289.

[ST1] John Stallings, Whitehead torsion of free products, Ann. of Math., 82 (1965), pp. 354-363.

[ST2] —_ Topologically unrealizable automorphisms of free groups, Proc. Amer. Math. Soc., 84 (1982), pp. 21-24.

[W1] F. WALDHAUSEN, Whitehead groups of generalized free products, in Lecture Notes in Math. 342, Springer-Verlag, New York, 1973, pp. 155-179.

[W2] _ Algebraic K-theory of generalized free products, Parts 1 and 2, Ann. of Math., 108 (1978), pp. 135-256. 
